\title{
Tabularia
}

TABULARIA Sources écrites des mondes normands médiévaux Guillaume de Volpiano : Fécamp et l'histoire normande | 2002

\section{La diffusion de la toponymie scandinave dans la Normandie ducale}

Scandinavian place-names in norman duchy

François de Beaurepaire

\section{OpenEdition}

Journals

Édition électronique

URL : http://journals.openedition.org/tabularia/1760

DOI : $10.4000 /$ tabularia. 1760

ISSN : 1630-7364

Éditeur :

CRAHAM - Centre Michel de Boüard, Presses universitaires de Caen

Référence électronique

François de Beaurepaire, "La diffusion de la toponymie scandinave dans la Normandie ducale », Tabularia [En ligne], Guillaume de Volpiano : Fécamp et l'histoire normande, mis en ligne le 22 juillet 2002, consulté le 20 avril 2019. URL : http://journals.openedition.org/tabularia/1760 ; DOI : 10.4000/ tabularia. 1760 


\title{
La diffusion de la toponymie scandinave dans la Normandie ducale \\ Scandinavian place-names in norman duchy
}

\author{
François de BEAUREPAIRE \\ Société de l'Histoire de Normandie \\ 128, rue Jeanne d'Arc, 76000 Rouen
}

Résumé:

En marge des relevés de noms de lieux d'origine scandinave présentés par les historiens et les linguistes, quelques réflexions méritent d'être formulées. On peut d'abord remarquer que certains appellatifs scandinaves se sont perpétués plus ou moins longtemps dans l'ancien parler régional (ainsi la londe, le londel, le thuit, le becquet, les hogues) et que les noms de lieux qui en sont dérivés peuvent être sensiblement postérieurs à l'établissement des Vikings; il en est de même des noms de personnes scandinaves inclus dans les noms de lieux et qui demeurèrent en usage pendant plusieurs générations. En ce qui concerne la diffusion géographique des noms de lieux scandinaves, on constate qu'ils s'appliquèrent de façon assez impérative aux paroisses ou aux domaines ruraux, qui perdirent leurs dénominations carolingiennes, tandis que certains noms mineurs, ceux des cours d'eau et de hameaux secondaires, ont mieux survécu. Enfin la grande question en suspens est celle du contraste entre l'abondance du legs toponymique des Vikings et le petit nombre de témoins qu'ils ont laissé dans le vocabulaire régional.

Mots-clés: Vikings, Danelaw, toponymie, domaines ruraux, paroisses, dialecte normand.

\begin{abstract}
:
Behind scandinavian place names known by historians and linguists, some observations have to be formulated. Scandinavian appellatives can be seen in the ancient regional dialect (so, la londe, le londel, le thuit, le becquet, les hogues) and place names issued from it, may be noticeably later than viking settlement; it is the case for scandinavian person names included in places names and which have been used for several generations. Concerning the question of geographic diffusion of scandinavian place names, we observe they have been generally given to parishes or rural estates, which lost their Carolingian designations, whereas some minor names (rivers, hamlets) better survived. Last but not least, the question is the contrast between the affluence of viking place names and the small number of the evidences which have survived in the regional vocabulary.
\end{abstract}

Keywords: Vikings, Danelaw, toponymy, rural estates, parishes, Norman dialect. 
L'arrivée des Vikings en Normandie fut à l'aube du X $\mathrm{X}^{\mathrm{e}}$ siècle l'évènement majeur des origines de cette province. Mais une grande imprécision règne sur les conditions dans lesquelles ils s'y établirent; les textes de cette époque n'apportent que des informations bien indigentes et les traces archéologiques de leur implantation sont très rares. Heureusement la toponymie régionale, riche d'éléments scandinaves, nous apporte des données irremplaçables; les historiens ${ }^{1} \mathrm{y}$ ont en effet noté la présence fréquente d'éléments d'origine scandinave tels que tot " domaine rural ", comme dans Yvetot, beuf " maison, agglomération », comme dans Elbeuf, bec " cours d'eau », comme dans Caudebec et Bolbec, fleur « estuaire, port » qui est présent dans Honfleur et Harfleur, et de plusieurs autres éléments que sont dalle "vallée", londe "bois", tuit "essart", torp "village", gatte "chemin", homme et hou, qui l'un et l'autre signifient une île ou un site au bord de l'eau. Leur répartition est en vérité fort inégale, car de fortes densités règnent dans le pays de Caux, la vallée de la Seine et les côtes du Cotentin, tandis qu'on ne trouve que des exemples dispersés dans le pays de Bray et qu'ils sont presque inexistants dans le sud de l'Eure et du Calvados et dans presque tout le département de l'Orne.

Les limites de notre propos ne nous permettent pas de présenter un inventaire détaillé du legs toponymique des Vikings en Normandie, même si nous restreignons notre investigation à l'ouest du pays de Caux, dont Fécamp est l'une des principales agglomérations. Nous nous limiterons à quelques observations générales que nous inspire le contraste manifeste entre la densité des noms de lieux d'origine scandinave et la pauvreté du legs linguistique de cette origine. Cette pauvreté découle des conclusions de l'investigation de Jean Renaud ${ }^{2}$, qui à la suite d'un large examen propose environ cent cinquante mots d'origine scandinave, qui relèvent d'ailleurs souvent de la pêche et de la navigation. Comment se fait-il donc que ces envahisseurs qui ont parrainé tant de noms de lieux en Normandie - n'y a-t-il pas plus de trois cents noms en - tot? - aient laissé si peu de témoins dans le parler régional? Sans prétendre donner de réponse satisfaisante à cette interrogation, nous chercherons du moins à proposer quelques réflexions qui orienteront les investigations des chercheurs futurs.

Nous tenterons d'abord de donner un aperçu de la toponymie du pays de Caux, telle qu'elle se présentait au $\mathrm{X}^{\mathrm{e}}$ siècle, lorsque les Vikings s'y établirent, et nous examinerons comment les noms de lieux de type scandinave y prirent place.

1. Le recensement des éléments scandinaves présents dans la toponymie normande, qu'on trouve dans les différents ouvrages historiques concernant la Normandie procède principalement de l'ouvrage de Charles JoRET, Des caractères et de l'extension des patois normands, étude de phonétique et d'ethnographie, Paris, F. Vieweg, 1883. XXXII-211 p. + cart. Une très bonne réflexion sur cette question nous a été récemment donnée par Gillian FELLOWS JONES, « Les noms de lieux d'origine scandinave et la colonisation viking en Normandie. Examen critique de la question ", Proxima Thulé, vol. 1, 1994, p. 63-103.

2. RENAUD, Jean, Les Vikings et la Normandie, Rennes, Ouest-France, 1989, 223 p. 
Le paysage toponymique y était alors constitué de plusieurs familles de noms de lieux plus ou moins largement représentées, issues de modèles prélatins, gallo-romains, germaniques et romans.

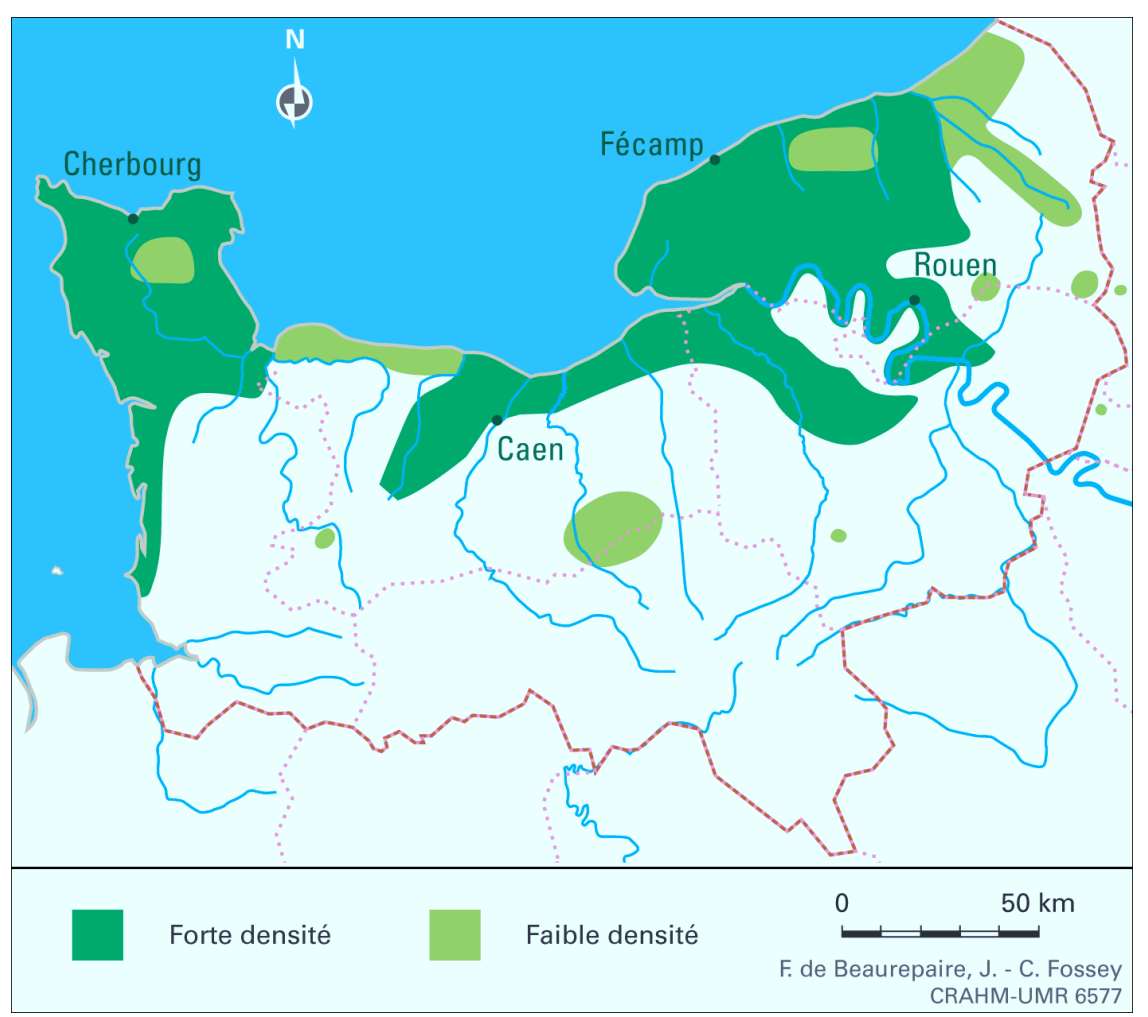

Les noms de lieux scandinaves en Normandie.

On note leur forte densité dans les régions côtières à l'exception de réserves correspondant aux massifs forestiers (notamment dans la Basse-Seine) ou à des points de résistance à l'implantation des Vikings. Dans l'arrière-pays leur présence se limite à quelques sites dispersés.

Les noms que nous présumons d'origine prélatine se rapportent soit à la langue gauloise, soit aux parlers qui l'ont précédée, sans qu'il soit possible de faire la distinction avec certitude, car il est probable que la langue gauloise avait ellemême intégré de notables éléments de langages plus anciens. On peut, comme ailleurs en France, considérer comme prélatins de nombreux noms de cours d'eau; la Seine représente, comme chacun sait, un primitif Sequana, témoin d'un passé très reculé; peut-être aussi anciens sont les noms des rivières du littoral cauchois, le Dun (Dunus), la Scie (Seda) la Saâne (Sedana) et son affluent la Vienne (Vigenna). En ce qui concerne les noms de lieux habités d'origine prélatine nous ne relevons que de rares exemples, Jumièges (Gemeticum), Rançon (Rosontio), 
Gravenchon (Craventio), Gueures (Gora) et L'eure (Lodurum), ancienne paroisse médiévale, aujourd'hui intégrée dans la ville du Havre. Lillebonne, ancien Juliobona, date, quant à lui, des premiers temps de la présence romaine, puisque l'appellatif gaulois bona « fondation » y est associé au nom d'homme bien latin Julius (Jules).

Mais le legs toponymique gallo-romain comprend surtout, comme ailleurs en France les héritiers de noms de domaine en acum ou iacum, dont le premier élément est le plus souvent un nom d'homme; ainsi Sabiniacum ou Pauliacum étaient les domaines de Sabinus et de Paulus avant de devenir les nombreux Savigny et Pouilly que nous connaissons. Or, les noms de lieux de ce modèle sont particulièrement peu nombreux dans le pays de Caux au regard de leur densité dans le reste de la France: on n'y compte que de rares noms de paroisse, bien que la structure paroissiale fût bien établie dans cette région à l'époque mérovingienne, ainsi que l'atteste le grand nombre d'églises dédiées de façon bien caractéristique aux saints alors en honneur, saint Rémi, saint Denis, saint Médard ou saint Martin ${ }^{3}$ : du type de formation toponymique en acum il ne subsiste guère dans le pays de Caux que les noms de paroisses de Drosay, le domaine de Drusus, de Luneray, le domaine de Lunaris, d'Autigny, formé avec le nom d'homme Altinus, Cany dérivant quant à lui d'un archétype indéterminé, ces noms étant par ailleurs groupés entre Saint-Valery-en-Caux et Dieppe. La survie des formations en acum fut plus fréquemment assurée dans les noms de hameaux: nous en relevons au moins une vingtaine, les noms d'hommes avec lesquels ils sont construits appartenant en général à la basse latinité ou à l'anthroponymie germanique diffusée en Gaule à partir des $\mathrm{V}^{\mathrm{e}} / \mathrm{VI}^{\mathrm{e}}$ siècles, comme le montrent les quelques exemples qui suivent:

Noms de hameaux formés avec des noms d'hommes de basse latinité

$\begin{array}{ll}\text { Bailly, à Yvetot } & \text { Ballius } \\ \text { Brilly à Vattetot-sous-Beaumont } & ? \\ \text { Clémencé à Manneville-ès-Plains } & \text { Clemens } \\ \text { Clercy à Bornambusc } & \text { Claricus } \\ \text { Gruchy à Montivilliers et Veulettes } & \text { Crucius } \\ \text { Reffigny à Yvetot } & \text { Rufinus } \\ \text { Tessy à Offranville } & \text { Tassius } \\ \text { Torcy à Ourville-en-Caux } & \text { Tauricius }\end{array}$

Noms formés avec des noms d'homme germaniques

Etupigny à Octeville

Stuppo

Gogny à Ecretteville-les-Baons

Godo

3. Beaurepaire, François de, "Essai sur le pays de Caux au temps de la première abbaye de Fécamp ", L’abbaye bénédictine de Fécamp, Ouvrage scientifique du XIII centenaire, t. I, Lecerf, Rouen, 1955, p. 3-21. 


$\begin{array}{ll}\text { Picagny à Bec-de-Mortagne } & \text { Piccho } \\ \text { Roquigny à Avremesnil } & \text { Rocco } \\ \text { Vinchigny à St-Martin-aux-Buneaux } & \text { Witso } \\ \text { Les trois Glatigny d'Auberville-la-Renault, } & \\ \text { Fontaine-la-Mallet et Sainte-Marguerite-sur-Duclair } & \text { Glatto }\end{array}$

A la même époque, alors que les formations en acum/iacum cessaient de se multiplier, apparut en Gaule une éclosion de noms de lieux empruntés aux langues germaniques. Bien que les migrations barbares aient surtout affecté le nord et le nord-est de la Gaule, leur pénétration a touché tout le pays, comme le montre un semis de toponymes de cette origine, dont les exemples régionaux s'appliquent à quelques lieux notables, Flers (Orne), Falaise (Calvados), Rebets, Meulers et Darnétal (Seine-Maritime), et notamment la ville de Fécamp. En effet, malgré la légende qui voit dans Fécamp un ancien Fici campus "le champ du figuier", comme le consacre le blason de la ville meublé d'un superbe figuier, il semble bien qu'il s'agit d'un nom d'origine germanique; en effet les formes anciennes contredisent formellement l'interprétation " champ du figuier ", notamment un diplôme de Charles le Chauve de 875 en faveur de la cathédrale de Rouen ${ }^{4}$ qui mentionne le lieu désigné Fontanas super fluvium Fiscannum: il s'agit visiblement de la rivière de Valmont qui traverse aujourd'hui la ville. Or Fiscannum est de toute évidence apparenté à la Fischa, affluent du Danube, et aux nombreux Fischach et Fischbach allemands, issus de la base européenne fisk " poisson »; par ailleurs un petit affluent de l'Aude, le Fresquel, est désigné en 835 sous la forme Fiscanum, bien proche de l'ancien nom de Fécamp, sa présence dans le sud de la France étant un témoin de la colonisation du Languedoc par les Wisigoths. En tout état de cause, il n'y a pas lieu de rechercher pour Fécamp une origine prélatine, puisque la lettre $F$ est absente de la langue gauloise.

En dehors de ces rares formations germaniques, la toponymie gallo-romaine céda surtout la place à partir du VI siècle aux formations romanes caractérisées par l'emploi d'une gamme d'appellatifs de valeur géographique, tels que val, mont, bosc ou bois, ou représentatifs de l'habitat humain, comme cour, ville, villiers et mesnil, les noms en ville le plus anciennement attestés en Normandie étant Bourville en Seine-Maritime (Bodardi villa 715) et Bazenville dans le Calvados (Basonni villa 875); les noms de lieux relevant de ce type de formation se sont dès lors multipliés jusqu'au XII ${ }^{\mathrm{e}}$ siècle, si bien qu'il est souvent difficile de savoir s'ils sont antérieurs à l'arrivée des Vikings, contemporains ou postérieurs.

4. Tessier, Georges, Recueil des actes de Charles II le Chauve, roi de France, Paris, Académie des Inscriptions et Belles-Lettres, 1943-1955 (3 vol.), III, p. 387. 
Après avoir recensé les modèles toponymiques qui préexistaient à la conquête des Vikings, nous avons à examiner comment et sous quelle forme se sont implantées les désignations scandinaves: celles-ci sont de différents modèles qu'il convient de présenter.

Parmi les formations scandinaves présentes en Normandie, les plus caractéristiques sont celles qui ont leurs équivalents dans les sites d'origine des Vikings, dans le Jutland ou dans le Danelaw, cette région du nord-est de l'Angleterre où ils s'étaient établis dès la seconde moitié du IX ${ }^{\mathrm{e}}$ siècle. Tel est le cas des Hautot, semblables aux Hottoft anglais, de Dieppedalle apparenté au Dibendal danois, des Bouquelon et Iclon, décalques des Bogelund et Egelund du Danemark, désignations de hêtraies et de chênaies; de même les Houlbec normands évoquent les innombrables Holbaek danois, tandis qu'Auppegard et Houlgate sont cousins germains des Applegarth et des Holgate du Danelaw.

A côté des composés toponymiques scandinaves qui ont pris place tels quels en Normandie sous réserve de quelques mutations phonétiques, certains autres ont été affectés par différentes formes de romanisation. Dans un premier cas de figure, ils ont subi la flexion du féminin pluriel roman, ainsi pour Boos (ancien Bothes), Tôtes, Veules, Ecalles (Scaloe) ou Etigue à Vattetot-sur-mer (Stigæ). Un autre formule fut celle de l'association d'un appellatif scandinave et d'un adjectif roman, ainsi dans Bonnetot, Maltot et Franquetot et aussi dans Belbeuf, près de Rouen, ancien Bellebeuf, ou dans Cléronde à Blay (Calvados) qui est un ancien Clairlonde.

La romanisation s'est aussi manifestée par la conjugaison d'appellatifs scandinaves avec des noms d'hommes romans ou avec des noms d'hommes d'origine germanique en usage en Gaule dans la seconde moitié du premier millénaire. Dans le premier cas on peut citer l'exemple de Mictot, hameau d'Angerville-laMartel, proche de Fécamp, anciennement "Mikieltot» "le domaine de Michel » ou de Barbetot à Epretot, où l'on identifie le nom d'homme roman Barbé ou Barbet. Les exemples de la seconde formule sont encore plus nombreux: citons seulement Robertot, Gratot, Raimbertot et Thiboutot, dans lesquels on reconnaît les noms d'homme Robert, Guérard, Raimbert et Thibout.

Enfin la dernière forme de romanisation fut celle de l'intégration des appellatifs toponymiques scandinaves dans la langue romane, impliquant l'emploi de l'article et parfois une suffixation. On les trouve surtout dans la microtoponymie; qu'il nous suffise d'évoquer tous les le bec, le becquet, la hogue, la hoguette, la londe, le londel, la londette, le homme, le hommet; ces formations se sont multipliées jusqu'à une date assez tardive, parfois jusqu'à la fin du Moyen Age et leur aire de diffusion a largement débordé celle de la première occupation scandinave, puisque nous retrouvons des lieux dits la Hogue dans le Maine et un Becquet près 
de Beauvais. De tels noms n'ont pas lieu d'être pris en considération pour définir les premiers secteurs d'établissement des Vikings.

Il n'y a pas lieu non plus de tirer un argument trop décisif de la présence de noms d'homme scandinaves dans les noms de lieu; en effet, parmi les noms d'homme portés par les Vikings ${ }^{5}$, les uns demeurèrent apparemment sans postérité et ne nous sont attestés qu'en construction toponymique, notamment dans des noms en tot ou en ville; mais certains autres bénéficièrent d'un vogue durable: on trouve des Asketillus, des Osmundus, des Turstingus, des Osulfus dans des chartes du XII ${ }^{\mathrm{e}}$ siècle et le succès des noms de famille Anquetil, Osmond, Toutain et Auzou témoigne de leur longue utilisation: les noms de lieux dans lesquels sont inclus, Ancretteville, Osmonville, Toutainville et Auzouville, ne sont donc pas totalement significatifs de la géographie des établissements des Vikings.

Dans la recension des noms de lieux scandinaves, il y a donc lieu de tenir compte de ce qu'un grand nombre de formations tardives correspondent à la longue survie de certains éléments toponymiques ou anthroponymiques scandinaves. Seuls les noms que l'on peut rapporter à la première heure de l'établissement des Vikings nous intéressent. En ne retenant que l'ouest du pays de Caux, on constate qu'ils y ont largement pris place pour la désignation de nombreux centres d'habitat, qu'il s'agît de simples paroisses comme Daubeuf et Criquebeuf, Yvetot, Sassetot ou Raffetot, ou même d'agglomérations plus importantes telles que Caudebec, Elbeuf ou Harfleur. Seuls échappèrent à de nouvelles désignations, comme nous l'avons vu, quelques noms de cours d'eau et d'agglomérations notables, Fécamp, l'Eure ou Lillebonne, ainsi qu'un petit groupe de paroisses entre Saint-Valery et Dieppe, mais aussi un semis de petits hameaux, dotés de noms gallo-romains en acum, comme si les Vikings s'étaient employés à donner de nouvelles désignations aux centres paroissiaux et aux sièges des grands domaines fonciers qu'ils s'étaient appropriés en domestiquant plus ou moins la population locale, avaient négligé de procéder de même pour les centres d'habitat secondaires, peuplés de paysans, qui purent conserver leurs toponymes traditionnels.

Ces remarques s'appliquent-elles aux autres régions de Normandie occupées par les Vikings? Nous pouvons répondre affirmativement pour le Cotentin qui fut très densément occupé par les Vikings. Nous constatons en particulier que dans les deux cantons de Beaumont-Hague et des Pieux nous ne retrouvons qu'un seul nom de lieu issu d'un nom gallo-romain en acum, le hameau de Gruchy à Gréville, tandis que tous les noms de paroisses sont contemporains de la conquête scandinave ou postérieurs à celle-ci.

5. Sur ce point il y a lieu de se référer à Jean ADIGARD des GAUTRIES, Les noms de personnes scandinaves en Normandie de 911 à 1066, C. Bloms Boktryckeri, Lund, 1954. 
Ces remarques n'ont cependant pas lieu d'être généralisées à tout le littoral normand, car le Bessin, entre la Vire et la Seulles, demeure peuplé d'un nombre appréciable de noms de lieux d'origine prélatine ou gallo-romaine, tels que Vienne, Ryes, Tracy, Sully ou Maisy; le Bessin contient même avec l'Avranchin une des plus fortes densités de noms de lieux antiques de Normandie. En revanche le vocabulaire agraire de cette région est curieusement enrichi d'un certain nombre de désignations de terroirs anglo-saxonnes, tels que delle, forlenc, hovelland, wendinc ou verlinc ${ }^{6}$, et l'on peut se demander si elles n'ont pas été diffusées par une main d'oeuvre venue de Grande-Bretagne à l'époque de l'établissement des Vikings. Ce schéma historique, bien différent de celui du pays de Caux et du Cotentin, montre combien l'établissement des Vikings en Normandie a pu connaître des aspects différenciés.

Et nous en venons à cette contradiction que nous avons déjà soulignée entre la pauvreté du legs dialectologique des Vikings et la richesse de leur héritage toponymique. Pour apprécier ce phénomène, il paraît intéressant d'examiner ce que furent dans ces deux domaines les effets des diverses migrations qui affectèrent le nord-ouest de l'Europe au cours du premier millénaire.

Nous pouvons en premier lieu porter notre regard sur les peuples issus de l'ancienne Germanie, qui s'établirent en Gaule au Ve siècle. Nous leur devons la généralisation de parlers germaniques au nord-est d'une courbe rejoignant la Flandre à la Suisse romande; la toponymie au delà de cette ligne est devenue dans son ensemble de type germanique, mais de notables témoins de la toponymie gallo-romaine y ont subsisté, principalement dans la vallée de la Moselle ${ }^{7}$. Dans tout le reste de la Gaule ces migrations ont donné naissance à des noms de lieux germaniques qui ne furent parfois qu'un saupoudrage, mais présentent en certaines régions une appréciable densité, ainsi en Bourgogne, où l'on recense de quelque deux cents noms construits avec le suffixe ans (issu du suffixe germanique ing), comme Ornans, Louhans ou Rémondans, et dans une moindre mesure dans le Languedoc où un groupe de noms en ens, tels que Rabastens, Badens ou Tonneins, évoquent l'ancienne colonisation wisigothe. Mais malgré le succès régional

6. Cf. Musset, Lucien, "Pour l'étude des relations entre les colonies scandinaves d'Angleterre et de Normandie ", Mélanges de linguistique et de philologie Fernand Mossé, Paris, Didier, 1959, p. 330-339.

7. HAUBRICHS, Wolfgang, "Le processus d'élaboration des frontières linguistiques: le cas des zones de contact romano-germaniques » et PITZ, Martina, " Le superstrat francique dans le nord-est de la Gaule », Nouvelle revue d'Onomastique, 35-36, 2000, p. 4185 . 
de ces formations, elles n'ont pas été accompagnées d'une survie des parlers germaniques en dehors de quelques éléments lexicaux.

Bien différentes furent les conséquences des migrations qui affectèrent la Grande-Bretagne. Les Anglo-Saxons, venus du Jutland, après avoir traversé la mer du Nord, y imposèrent à la fois leur langue et leurs désignations toponymiques, balayant presque toute la toponymie celtique préexistante à l'exception des noms de cours d'eau. A leur tour les Bretons pourchassés allèrent s'installer massivement en Armorique, notre actuelle Bretagne, et là aussi imposèrent leur langue aux populations locales (ce qui fut peut-être facilité par le fait qu'elles utilisaient peut-être encore un dialecte gaulois) et parallèlement diffusèrent dans cette province une toponymie spécifique, en éliminant presque toute la toponymie antérieure.

Tout autres furent les conséquences de l'installation des Vikings dans le nordest de l'Angleterre à la fin du IX' siècle, peu avant leur descente en Normandie. Ils ont peuplé tout ce secteur géographique d'une abondante toponymie scandinave apparentée à celle de la Normandie; en revanche la langue des Vikings n'a guère laissé plus de traces dans la langue anglaise que dans le langage régional normand.

Et pour compléter notre tour d'horizon, il nous intéresse de porter notre regard sur les conséquences de l'établissement des compagnons de Guillaume le Conquérant en Angleterre en 1066, dans les deux domaines de la linguistique et de la toponymie. Il eût été normal que les Normands, à la faveur de leur position dominante, apportassent au paysage toponymique de l'Angleterre de nombreuses nouvelles désignations nouvelles empruntées à la langue romane: or, sauf quelques exceptions (ainsi Montacute, Richmond ou Freemantle) ${ }^{8}$, ils s'en abstinrent, bien que leurs descendants n'aient cessé d'employer jusqu'au $\mathrm{XV}^{\mathrm{e}}$ siècle un parler dérivé du langage régional normand, dont l'apport à la langue anglaise demeure considérable.

On peut s'interroger sur les raisons qui ont dicté la présence ou l'absence de corrélation entre les créations toponymiques et les conséquences linguistiques des invasions du premier millénaire? Dans certains cas les envahisseurs ont imposé la diffusion de leur langue, mais respecté la toponymie locale; ailleurs, ainsi en Normandie, le langage régional s'est maintenu, alors que les noms de lieux préexistants étaient largement éliminés au profit d'appellations nouvelles. Des facteurs d'ordre politique, démographique ou sociologique expliquent sans doute ce paradoxe; puissent les historiens futurs réussir à les mettre en lumière avec prudence et perspicacité.

8. Cf. Ekwall, Eilert, The concise Oxford dictionary of english place-names, Oxford, 1947, p. XXV-XXVI. 